\title{
Inertial and Gravitational Mass in General Relativity and Their Cosmological Consequences
}

\author{
Miguel Portilla \\ Departament d'Astronomia i Astrofísica, Universitat de València, València, Spain \\ Email: miguel.portilla@uv.es
}

How to cite this paper: Portilla, M. (2021) Inertial and Gravitational Mass in General Relativity and Their Cosmological Consequences. Journal of Modern Physics, 12, 1729 1748.

https://doi.org/10.4236/jmp.2021.1213101

Received: July 16, 2021

Accepted: November 1, 2021

Published: November 4, 2021

Copyright (C) 2021 by author(s) and Scientific Research Publishing Inc. This work is licensed under the Creative Commons Attribution International License (CC BY 4.0).

http://creativecommons.org/licenses/by/4.0/ (c) (i) Open Access

\begin{abstract}
We revise the concept of mass of a particle in general relativity initiated by Einstein, Brans, and Rosen in the fifties, using the results of P. Havas and J.N. Goldberg on the equations of motion for point-like particles. We show how one can define a constant inertial mass, and a variable gravitational mass dependent on their gravitational interaction with the rest of particles. The introduced gravitational mass allows us to construct a cosmological model that satisfactorily accounts for the observed deficit of mass, the dark energy and the cosmological constant, without the assumption of new forms of matter or energy: dark matter and dark energy can be explained as a gravitational effect in the framework of the standard general theory of relativity.
\end{abstract}

\section{Keywords}

Dark Matter, Dark Energy, Cosmological Constant: Gravitational Mass

\section{Introduction}

We interpret in this paper the cosmological dark matter and energy present in the epoch of galactic dominance as a pure gravitational effect, using primarily baryonic matter and standard general relativity.

In a Minkowski spacetime, a fluid is the result of a statistical average of the dynamical properties of a system of particles; in a curved space, as it is the case when the particles are self gravitating, one needs also to revise the concept of mass. The distinction between inertial mass and active and passive gravitational mass and its relation with the weak equivalence principle was thoroughly compiled in Jammer's book [1], of which we give a few details in the next section. The revision in the framework of general relativity will permit to explain the dark components present in the galactic dominant epoch. We shall use the metric generated by a finite system of particles, i.e., a solution of the Einstein's equ- 
ations, having as source a distribution with support on the world lines of the particles. In the sixties, P. Havas and J.N. Goldberg [2] developed a method of successive approximations to find it. They used harmonic coordinates, unique up to Lorentz transformations [3] [4], and an auxiliary Minkowski tensor $\eta$, defined as the one that in harmonic coordinates verifies $\eta(a, b)=\eta_{\mu \nu} a^{\mu} b^{\nu}$, with $\eta_{\mu v}=\operatorname{diag}(1,-1,-1,-1)$, for any pair of vectors. Serious problems of convergence over the particle world lines were easily overcome in the first approximation [2], and needed of more efforts in the second one [5]. These results were useful to interpret in the eighties the first indirect proves of gravitational radiation by compact bodies [6]. Fortunately, we shall have enough with the first approximation.

There is some rejection to consider point-like particles (the use of unidimensional Dirac distribution as source), due to the problems of convergence, predicted by a theorem due to Geroch-Traschen [7] that states a necessary condition for the existence of regular metrics (locally bounded, with locally square integrable weak first derivative), by requiring the support of the distributions to be tridimensional. However, Katanaev [8] solved exactly the problem in the case of only one point-like particle, obtaining, as hoped, a non regular metric in the sense of Geroch-Traschen, with an additional coordinate singularity that makes necessary a metric extension. Recently, we have obtained [9] a maximal extension of this metric that is regular and has, as hoped, a distribution with tridimensional support as source, in accordance to the Geroch-Traschen theorem. Unfortunately, these results has not been extended to more than one particle, and we must use the approximated solutions [2] [5].

Following Havas-Goldberg (H-G), we consider the spacetime as a manifold provided with two tensors fields $(M, g, \eta)$ : the first is the true metric, generated by point-like particles, and the second is an auxiliary tensor used to obtain the true metric. In the next section, we show that the paper by H-G [2] tacitly contains valuable definitions for a constant inertial and a time dependent passive gravitational mass, though the last one, inexplicably, was not presented as such. We shall show that their quotient, though being the same for all the particles, is not a constant, because the passive gravitational increases with the particle's proper time, impeding the equality of inertial and gravitational mass and suggesting that their difference might account for both dark components. Section 3 summarizes the dynamical properties of a cosmological fluid made of self gravitating particles obtained recently by us [10], and Section 4 shows how the gravitational mass introduced in this paper explains satisfactorily both dark matter and dark energy.

\section{Inertial and Passive Gravitational Mass of Point-Like Particles}

Classical physics distinguishes three kinds of mass: inertial and, active and passive gravitational mass; but these concepts change when we move from Newto- 
nian mechanics to special and general relativity.

Newtonian mechanics identifies the three masses, and links them to the law of motion as proportionality between force and acceleration or, alternatively, to the law of conservation of the total tri-momentum: given an isolated system of $N$ interacting particles there exist $N$ real numbers such that the sum $\sum_{a=1}^{N} m_{a} \frac{\mathrm{d} x_{a}}{\mathrm{~d} t}$ is constant [1] [11].

Special relativity distinguishes the proper inertial mass (or rest mass), related to the conservation of the total-four-momentum (where the absolute Newtonian time $t$ is substituted by the particle's proper time $\tau_{a}$ ), and the relativistic mass, that in a general inertial frame has the familiar velocity dependence $m_{a} / \sqrt{1-v_{a}^{2}}$.

General relativity, as reported in Jammer's book [1], has not yet achieved general agreement with the distinction between inertial mass and active and passive gravitational mass:

Einstein in 1950 [12], using a weak field approximation to describe the metric due to a finite distribution of matter, concluded that the inertial mass of a body, identified with the gravitational mass by principle, is not constant because depends on its gravitational interaction with all the other present masses.

C.H. Brans in 1962 [13] by studying the motion of a test particle in the field of a massive particle, at rest at the centre of a shell with mass $M_{S}$ simulating the effect of the universe, contrarily argued the equality of inertial and active gravitational mass, and its independence of the gravitational interaction, at variance with the Mach's principle. N. Rosen in 1965 [14] replied analyzing the equation of motion of a test particle in the field produced by a more massive particle, immersed now in an true expanding universe, obtaining a variable inertial mass (equal to the passive gravitational mass) which increases as the universe expands, and a constant active gravitational mass. So, in short: Rosen partially extended the Einstein's result to a cosmological setting, obtaining a variable inertial mass equal to the passive gravitational mass; and coinciding with Brans about the constancy of the active gravitational mass.

H.C. Ohanian in 2013, [15] defined the inertial mass of an object in an asymptotically flat spacetime as the volume integral of an energy density determined by the canonical energy-momentum tensor, and its gravitational mass by the asymptotically Newtonian potential at large distance from it; and, he also demonstrated the equality of them. But asymptotic flat conditions are not satisfied in cosmology.

The concept of mass varies when we move from Newtonian mechanics to special relativity and to general relativity.

Not all the authors have considered that in general relativity might exist different definitions of mass. Rosen clearly stated that his definition of inertial mass, based on an interpretation of the equation of the geodesics, is not the only possible [14]. The aforementioned Einstein, and Rosen models, were developed under the tacit assumption of the Mach's principle: the inertial mass of a body is due to its interaction with all the other masses in the universe. Brans abandoned 
this principle in his critic of these models.

In this paper we also contradict the Mach's principle by considering a constant inertial mass, and identical active and passive mass depending on the gravitational interaction. A satisfactory extension to general relativity of the inertial mass of a point-like particle can be proposed, in the framework of the H-G work [2], by linking the concept directly to the Einstein's field equations (in this way it is linked also to the equations of motion). To follow the process it will be convenient to recall the two world line parametrizations used by H-G, namely, the physical proper time $\mathrm{d} s=\sqrt{g_{\alpha \beta} \mathrm{d} z^{\alpha} \mathrm{d} z^{\beta}}$, and the Minkowski proper time $\mathrm{d} \tau=\sqrt{\eta_{\alpha \beta} \mathrm{d} z^{\alpha} \mathrm{d} z^{\beta}}$. We shall denote by $u_{a}^{\mu}=\frac{\mathrm{d} z^{\mu}}{\mathrm{d} s_{a}}$ and $v_{a}^{\mu}=\frac{\mathrm{d} z^{\mu}}{\mathrm{d} \tau_{a}}$ the tangent vectors to the world line of the particle " $a$ ", corresponding to both parametrizations. Consequently, $g\left(u_{a}, u_{a}\right)=1, \eta\left(v_{a}, v_{a}\right)=1$. From the first equation one gets

$$
\frac{\mathrm{d} \tau_{a}}{\mathrm{~d} s_{a}}=\frac{1}{\sqrt{g\left(v_{a}, v_{a}\right)}}, g\left(v_{a}, v_{a}\right)=g_{\mu v}\left(x\left(\tau_{a}\right)\right) v_{a}^{\mu} v_{a}^{v}
$$

We propose to define the particle's inertial mass $m_{a}$ and enunciate at once the field equations for a system of point-like particles:

Given a system of $N$ self-gravitating particles there exist $N$ real numbers $\left\{m_{a}, a=1, \cdots, n\right\}$, such that the metric $g$ of the spacetime is a solution of the Einstein's equations, with a distribution with support on the world lines of the particles as energy-momentum tensor.

$$
G^{\mu v}(g)=8 \pi G \sum_{a=1}^{N} \int m_{a} u_{a}^{\mu} u_{a}^{v} \delta^{(4)}\left(x-z_{a}\left(s_{a}\right)\right) \mathrm{d} s_{a}, u_{a}^{\mu}=\frac{\mathrm{d} z_{a}^{\mu}}{\mathrm{d} s_{a}} .
$$

We shall justify bellow to denote inertial mass to these constants. In order to define the passive gravitational mass we consider the particle's four-momentum $p_{a}=m_{a} u_{a}, a=1,2, \cdots, N$. By a simple calculation:

$$
\begin{gathered}
p_{a}^{\mu}=m_{a} u_{a}^{\mu}=m_{a} \frac{\mathrm{d} \tau_{a}}{\mathrm{~d} s_{a}} \frac{\mathrm{d} z^{\mu}}{\mathrm{d} \tau_{a}}=m_{a} \frac{\mathrm{d} \tau_{a}}{\mathrm{~d} s_{a}} v_{a}^{\mu} \text {, and substituting (1) we obtain } \\
p_{a}^{\mu}=M_{a}\left(\tau_{a}\right) v_{a}^{\mu} \text {, with } M_{a}\left(\tau_{a}\right) \text { related to } m_{a}: \\
M_{a}\left(\tau_{a}\right)=\frac{m_{a}}{\sqrt{g\left(v_{a}, v_{a}\right)}}
\end{gathered}
$$

coinciding with equation (16) in [2]. One can now interpret physically the constants $m_{a}$ and the functions $M_{a}\left(\tau_{a}\right)$ using the equations of motion derived by Havas-Goldberg from the null divergence of the energy momentum tensor (Equation (14) in [2]):

$$
\frac{\mathrm{d} p_{a \mu}}{\mathrm{d} \tau_{a}}=F_{a \mu}, F_{a \mu}=\frac{1}{2} M_{a}\left(\tau_{a}\right) \frac{\partial\left(g\left(v_{a}, v_{a}\right)\right)}{\partial x_{a}^{\mu}}
$$

that can be rewritten as:

$$
m_{a} \frac{\mathrm{d} u_{a \mu}}{\mathrm{d} \tau_{a}}=M_{a}\left(\tau_{a}\right) \frac{\partial U_{a}}{\partial x_{a}^{\mu}}, U_{a}=\frac{1}{2} g\left(v_{a}, v_{a}\right)
$$


to the equation of motion of a particle in special relativistic mechanics, justifying in this way to consider the constants $m_{a}$ as the inertial masses, and prompting us to interpret the functions $M_{a}\left(\tau_{a}\right)$ given in (3) as the passive gravitational masses of the particles. The aim of this paper is to show that this gravitational mass can explain the dark components and the cosmological constant (Inexplicably, H-G [2] did not used this concept).

Let us outline that the equations of motion (4) or (5) (suppressing subindex $a$ for the sake of simplicity) are equivalent to the geodesic equations. The geodesics equations generated by the Lagrangian $L=g_{\alpha \beta} \dot{x}^{\alpha} \dot{x}^{\beta}$, where dots denote derivatives respect the proper time are $\frac{\mathrm{d}}{\mathrm{d} s}\left(g_{\mu \nu} \dot{x}^{v}\right)=\frac{1}{2} \frac{\partial g_{\alpha \beta}}{\partial x^{\mu}} \dot{x}^{\alpha} \dot{x}^{\beta}$. Now, inserting the convector $p_{\mu}=g_{\mu \nu} p^{v}$ we can write: $\frac{\mathrm{d} p_{\mu}}{\mathrm{d} s}=\frac{m}{2} \frac{\partial g_{\alpha \beta}}{\partial x^{\mu}} \dot{x}^{\alpha} \dot{x}^{\beta}$, and changing the physical proper time to the Minkowski proper time we get the Equation (4) $\frac{\mathrm{d} p_{\mu}}{\mathrm{d} \tau}=\frac{1}{2} m \frac{\mathrm{d} \tau}{\mathrm{d} s} \frac{\partial g_{\alpha \beta}}{\partial x^{\mu}} v^{\alpha} v^{\beta}=\frac{1}{2} M(\tau) \frac{\partial g_{\alpha \beta}}{\partial x^{\mu}} v^{\alpha} v^{\beta}$.

The question arises if the definitions of mass given in Equations (2), (3) satisfy the weak equivalence principle (WEP). The answer is affirmative. In terms of the Hammer's book [1], pg. 103, the WEP for a point-like particle is stated as follows: "the world line of a particle, released at an initial space-time event with a given velocity is independent of the weight" (it means the passive mass).The WEP defines thus a preferred set of geodetic curves, as we have proven above.

Let us add a comment to the Jammer's distinction of two versions of WEP: the kinematic $\mathrm{WEP}_{\text {kin }}$, states that at a given location all bodies fall with the same acceleration (principle of universality of free fall), and the dynamic $\mathrm{WEP}_{\mathrm{dyn}}$ states that the ratio $m_{p} / m_{i}$ is the same for all the particles, or in appropriate units $m_{i}=m_{p}$.

When considering the torsion balance used in Eötvös-like experiments, the quotient $M_{a} / m_{a}=1 / \sqrt{g_{00}}$ is a constant, and the same for any of the two small masses of the balance, because $g_{00}$ corresponds to the metric produced by a static earth. So, in this particular case the $\mathrm{WEP}_{\mathrm{dyn}}$, in the sense of identity of inertial and gravitational mass, is fulfilled.

However, in subsection IIIC, where we study the Milne's universe with mass and prove the equality active and passive gravitational mass, we shall find that the quotient $M_{a}\left(\tau_{a}\right) / m_{a}$ is the same for all the particles, and therefore all them with the same initial conditions move equal in the gravitational field in accord with the $\mathrm{WEP}_{\text {kin }}$, but the quotient of masses is a monotonous increasing function of the expansion factor (see Equation (23)) and the $\mathrm{WEP}_{\text {dyn }}$ does not imply the equality of inertial and gravitational mass by choosing appropriated units. In section IV we shall develop, using the gravitational mass concept, far reaching cosmological consequences.

\section{A Cosmological Model Built with Point-Like Particles}

In this section we study two properties of the gravitational mass: the equivalence between active and passive gravitational mass, and finally their time dependence 
presented in Equation (18); but, the main objective is to construct a cosmological model composed of self-gravitating point-like particles. A cosmological model is a spacetime locally isotropic everywhere respect a cosmological observer [16], whose main characteristics we summarize in the next subsection with notations that will be useful to describe both, the standard model with dark components, and our model based uniquely on the gravitational mass defined in the previous section. It will be useful to start recalling the Milne's universe without mass, described in [16], and then to construct a Milne's universe with mass in section IIIC.

\section{1) The FRLW cosmological models}

A cosmological model may be characterized by an index of curvature $(k=1,0$, $-1)$, and giving the energy density $\rho_{F}(a)$ as function of the expansion factor, that will be convenient to write in the form

$$
\rho_{F}(a)=\frac{3 H_{0}^{2}}{8 \pi G}\left(\frac{\Omega_{M}}{a^{3}}+f(a)\right),
$$

with $H_{0}=\dot{a} /\left.a\right|_{a=1}$ and $\Omega_{M}+f(1)=1$. In the current cosmological model the first and second summands describe the unknown pressure-less dark matter and dark energy densities respectively; the cosmological constant $\Lambda$ may be considered as an important particular case of dark energy, with $f(a)=\frac{\Lambda}{3 H_{0}^{2}}$, because a successful cosmological model, the $\Lambda C D M$ model, has been developed with this assumption [17]. The model presented in this paper with a non constant $f(a)$ is equally successful as we show in IV; but, it has the advantage that the dark components may be accounted for with the gravitational mass concept. The Friedmann equation:

$$
\frac{\mathrm{d} a}{\mathrm{~d} s}=H_{0} a \sqrt{\frac{\Omega_{M}}{a^{3}}+\frac{\Omega_{k}}{a^{2}}+f(a)}, \Omega_{k}=-\frac{k}{H_{0}^{2} R_{0}^{2}},
$$

determines the expansion factor as function of the cosmological time $s$. Henceforth we shall take $k=0$. Taking into account the equation of continuity $\dot{\rho}_{F}+3 H\left(\rho_{F}+p\right)=0$, and well known calculations, one obtains the pressure and acceleration of the model:

$$
\begin{gathered}
p_{F}(a)=\frac{3 H_{0}^{2}}{8 \pi G}\left(-f(a)+\frac{1}{3} a \frac{\mathrm{d} f}{\mathrm{~d} a}\right) \\
\frac{\ddot{a}}{a}=-\frac{4 \pi G}{3}\left(\rho_{F}+3 p_{F}\right)=-H_{0}^{2}\left(\frac{\Omega_{M}}{2 a^{3}}-f(a)+\frac{1}{2} a \frac{\mathrm{d} f}{\mathrm{~d} a}\right)
\end{gathered}
$$

In the $\Lambda$ CDM model, $\Omega_{M}$ is the sum of the baryonic and dark matter density fractions: $\Omega_{M}=\Omega_{b a}+\Omega_{d m}$; in our model $\Omega_{M}=\Omega_{b a}(1+\alpha)$ and, accordingly with the interpretation of $\alpha$ we shall give bellow in Equation (13), $\frac{3 H_{0}^{2}}{8 \pi G} \frac{\Omega_{b a} \alpha}{a_{i}^{3}}$ is the gravitational mass density present at the beginning of the galactic dominance epoch, due to the gravitational interaction of the particles that collapsed to form the galaxies in the precedent epoch with $a<a_{i}$. 


\section{2) The Milne's universe}

It is a portion of the Minkowski spacetime formed by a future half-light cone filled with a set of straight time like lines concurrent on its vertex. The world lines are the integral curves of the cosmological observer, and their points of intersection with any hyperboloid of constant proper time $\tau$ define an homogeneous Poisson process, with constant number density equal to $N / \tau^{3}$, being $N$ a constant; however, the number density of the intersections with the hypersurface of inertial coordinate $t=$ constant is not uniform: $N t\left(t^{2}-r^{2}\right)^{-2}$. The change of coordinates $t=\tau \cosh \Psi, r=\sinh \Psi$ produces the metric for the Milne's universe $\mathrm{d} s^{2}=-\mathrm{d} \tau^{2}+\frac{1}{\tau^{2}}\left(\mathrm{~d} \psi^{2}+\sinh ^{2} \psi\left(\mathrm{d} \theta^{2}+r^{2} \sin ^{2} \theta \mathrm{d} \psi^{2}\right)\right)$. That is a cosmological model without mass and therefore with null energy-momentum tensor.

\section{3) The Milne's universe with mass}

We consider now the concurrent straight lines of the Milne's universe as world lines of particles (galaxies) with equal inertial mass $m$, and use the metric obtained by H-G [2] at first order in $G$ in harmonic coordinates. To describe the recent epoch of galactic dominance we must take into account the evolution of the universe from small perturbations to bigger condensations of matter. We shall do that, with extreme simplification, by assuming that the galaxies began to dominate at some initial Minkowski proper time $\tau_{i}$. For times $\tau<\tau_{i}$ the matter content was dominated by other particles ("first stars") that finally did aggregate to form the galaxies of the more recent universe. To complete the Einstein's equations the energy momentum tensor is written in the form of a distribution with support over the world lines of the particles, parametrized with the Minkowskian proper time, as given by H-G in Equations (29) and (13) of [2]

$$
\sqrt{-g} T^{\mu \nu}=\sum_{a=1}^{N} \int_{\tau_{i}}^{\tau_{0}} M_{a}\left(\tau_{a}\right) v_{a}^{\mu} v_{a}^{v} \delta^{(4)}\left(x-z_{a}\left(\tau_{a}\right)\right) \mathrm{d} \tau_{a}, v_{a}^{\mu}=\frac{\mathrm{d} z_{a}^{\mu}}{\mathrm{d} \tau_{a}} .
$$

We have denoted with a subindex the different particles, though all the inertial masses are equal: $m_{a}=m$. The quantity $M_{a}\left(\tau_{a}\right)$ is the passive gravitational mass introduced in the previous section by the Equation (3).

The energy density $\rho(x)$ of the fluid formed by the system of interacting particles can be obtained as result of the action $\left(\rho_{p p}, \phi_{x}\right)$ of the point particle distribution $\rho_{p p}=\sqrt{-g} T^{\mu v} u_{\mu} u_{v}$ over a convenient test function $\phi_{x}$ defined as follow: let $A$ be the neighbourhood of the point $x$ represented in Figure 1, defined by two neighbour hypersurfaces: $\tau=t+\Delta t / 2$ (that are also hypersurfaces of constant proper time: $s=s(t)+\Delta s / 2, s=s(t)-\Delta s / 2)$ and a thin time like cone; and let $S$ be the intersection of the hypersurface $\tau=t$ with the neighbourhood $A$. We choose as test function $\phi(x)$ the characteristic function of the set $A$ : $\phi_{x}(u)=1$ if $u \in A, \phi_{x}(u)=0$ otherwise. One can prove [16], by averaging over the ref line $L$, that

$$
\rho(x)=\lim _{\Delta t \rightarrow 0} \frac{\left(\sqrt{-g} T^{00}, \varphi_{x}\right)}{\operatorname{Vol}^{(3)}(S) \Delta t}=n(t) M(t)
$$




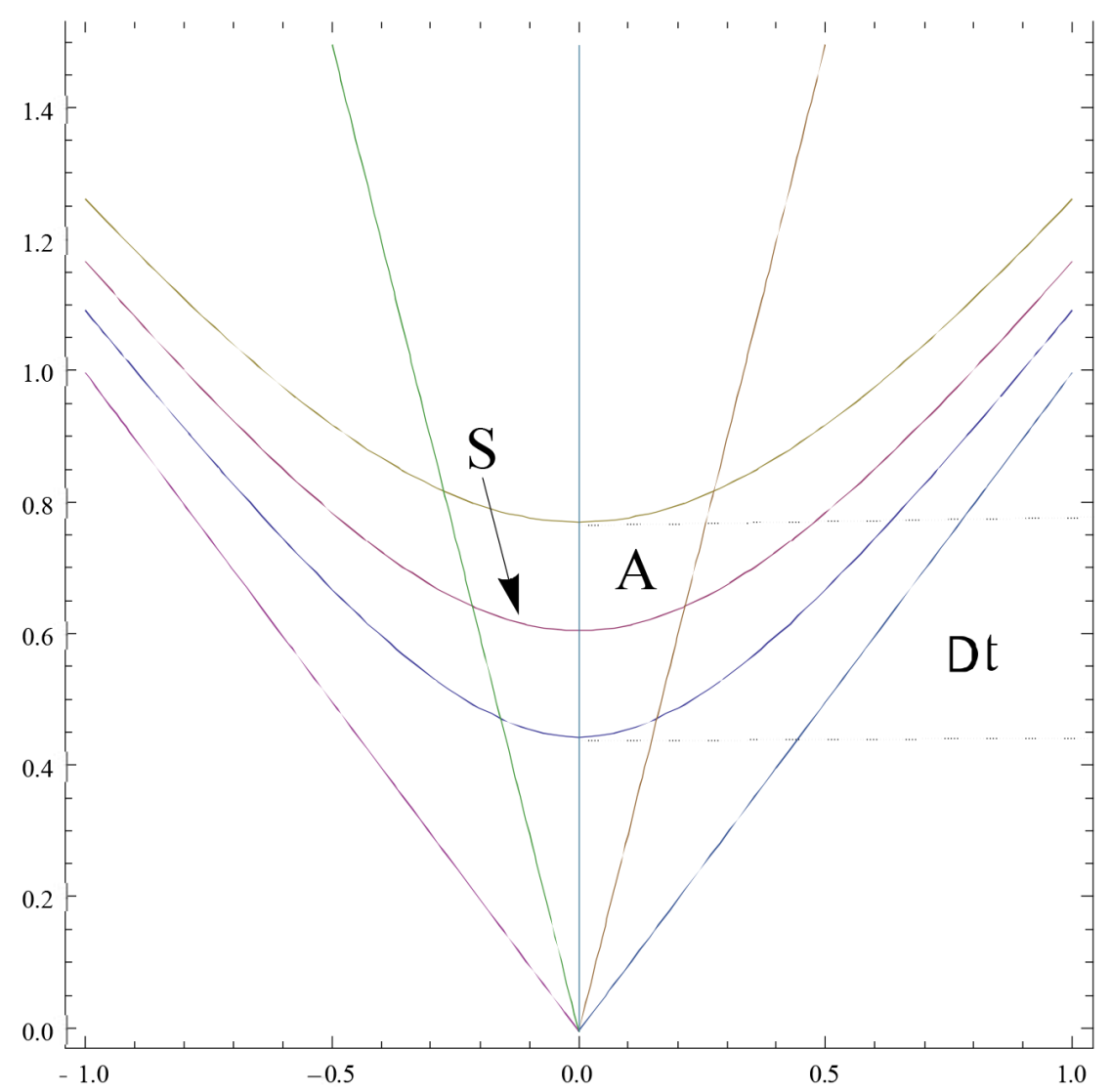

Figure 1. Element of volume $A$ centered at a point over a reference world line, limited by two surfaces $\tau=$ const. in a Milne's universe. One shows the tridimensional surface $S$ necessary to estimate the mass density.

Therefore $M(t)$ is also the active gravitational mass. This result proves that passive and active gravitational mass coincide, because according to equation (5), $M(t)$ was the passive gravitational mass. Henceforth we shall refer to $M(t)$ as the gravitational mass.

The iterative method involving a power series in $G$ developed in [2] allows to determine the metric and the gravitational mass in the form of two functional series: $g_{\alpha \beta}=\eta_{\alpha \beta}+g_{\alpha \beta}^{(1)}+\cdots$, and $M_{a}\left(\tau_{a}\right)=m_{a}+M_{a}^{(1)}\left(\tau_{a}\right)+\cdots$. A regularization is necessary at each order of approximation, because the metric diverges over the world lines of the particles. In our case, a great simplification is that in harmonic coordinates the world lines of the particles are still straight lines, despite the fact of having mass. The solution at first order in $G$ obtained by H-G (eq. 62 and 46) is the following:

$$
\begin{gathered}
g_{\mu \nu}^{(1)}\left(z_{a}(\tau)\right)=-4 G \sum_{b \neq a} \frac{m_{b}\left(\eta_{\mu \alpha} \eta_{\nu \beta} v_{b}^{\alpha} v^{\beta}-\frac{1}{2} \eta_{\mu v}\right)_{r e t}}{\eta\left(x-z_{b}, v_{b}\right)_{r e t}} \\
M_{a}^{(1)}\left(\tau_{a}\right)=-\frac{1}{2} m_{a} g_{\alpha \beta}^{(1)} v^{\alpha} v^{\beta}+m_{a} \alpha
\end{gathered}
$$

where in Equation (13) we have written as $m_{a} \alpha$ the constant of integration noted 
as ${ }_{2} C_{i}$ by $\mathrm{H}-\mathrm{G}$ in their Equation (46).

We interpret it as the gravitational mass present at time $\tau_{i}: m_{a} \alpha=M_{a}^{(1)}\left(\tau_{i}\right)$, i.e., as the gravitational mass acquired by the particles that collapsed during the anterior phase to form a galaxy in the present epoch; and bellow, in subsection IIIC1, this quantity will be identified with the detected dark matter.

As all the world lines of the Milne's universe are equivalent, by the Lorentz invariance of the equations, we shall obtain the physical metric and the gravitational mass over a world-line of reference $L: x^{\alpha}(t)=(t, 0,0,0)$, with null three-velocities $v^{k}=0$. Over this line the initial Minkowskian proper time $\tau_{i}$ $\left(\tau_{i}^{2}=t_{i}^{2}-\left|x_{i}\right|^{2}\right)$ coincides with the initial harmonic time $t_{i}$. The gravitational mass at a point $p=\left(t>t_{i}, 0\right) \in L$ is $M(t)=m \alpha+m\left(1-\frac{1}{2} g_{00}^{(1)}(p)\right)$, with

$$
g_{00}^{(1)}(p)=-G \sum_{b \neq a} \frac{m_{b}\left(\gamma_{b}^{2}-1 / 2\right)}{r_{b} \gamma_{b}\left(1+\bar{v}_{b}\right)}, \bar{v}_{b}=\frac{r_{b}}{t}, \gamma_{b}=\frac{1}{\sqrt{1-\left(\bar{v}_{b}\right)^{2}}} .
$$

The number of summands is finite even if the universe is made of infinite particles because only intervene the world lines intersecting the part of the past light cone of the point $p$ limited by the hyperboloid $\Sigma_{\tau_{i}}$, as shown in Figure 2. The

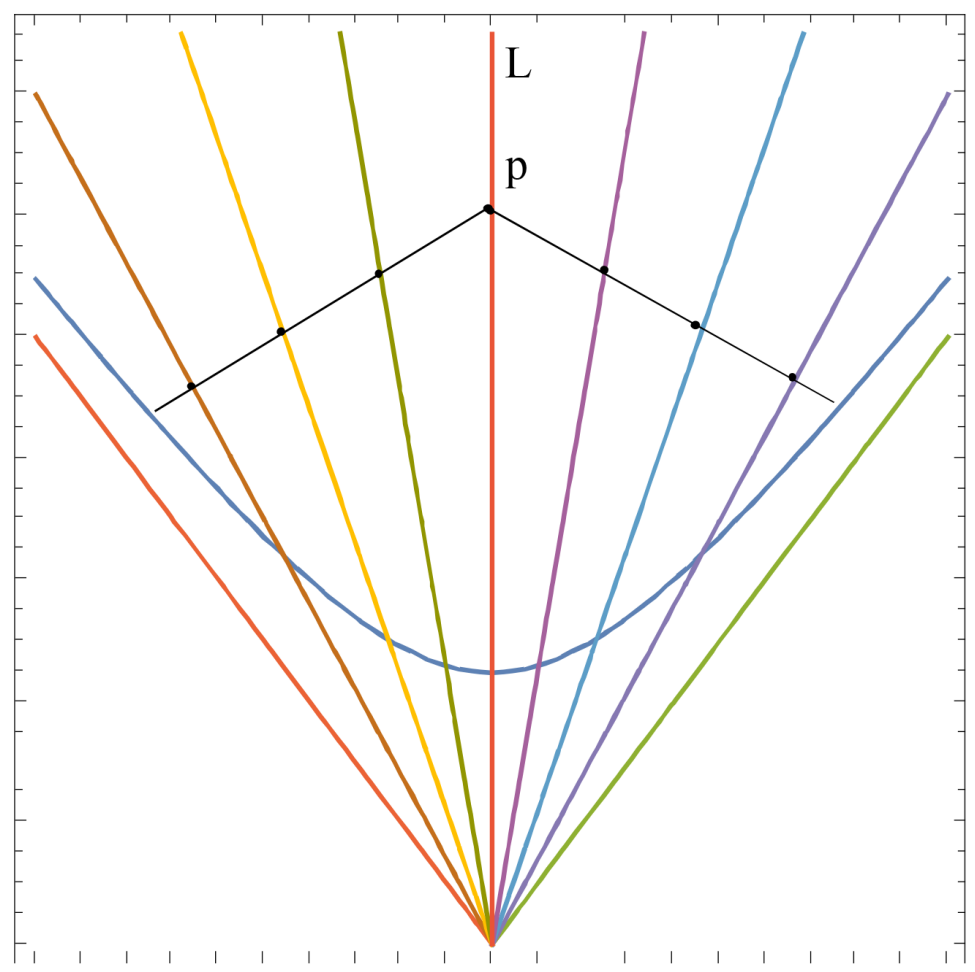

Figure 2. The past light cone at the point $p=(t, 0), t>\tau_{i}$ on the reference world-line $L$ intersects a finite number of world lines, limited by the hyperboloid $\tau=\tau_{i}$. When $t$ increases the number of particles contributing to its gravitational mass augments, and as a result thereof, an increment of their gravitational mass. The cosmological expansion increments the volume enclosing a given number of particles in a way (described in section IVA) that the gravitational mass density rapidly tends to a constant value, that we shall identify with the cosmological constant. 
sum in Equation (14) was estimated by integration in [10], hence we can obtain the metric over the reference line $L$ for $t>t_{i}$, and the ratio $\frac{\mathrm{d} t}{\mathrm{~d} s}=\frac{1}{g(v, v)}$ up to first order in $G$ :

$$
\begin{gathered}
\frac{\mathrm{d} t}{\mathrm{~d} s}=1-\frac{1}{2}\left\langle g_{00}^{(1)}(t)\right\rangle=1+\frac{\pi G N m}{3 t_{i}^{3}} t^{2} g\left(t, t_{i}\right) \\
\left\langle g_{00}^{(1)}(t)\right\rangle=-\frac{2 \pi G N m}{3 t_{i}^{3}} t^{2} g\left(t, t_{i}\right), g\left(t, t_{i}\right)=\left(1+\frac{t_{i}^{2}}{t^{2}}\right)-4 \frac{t_{i}^{2}}{t^{2}}\left(\frac{3}{2}\left(1+\frac{t_{i}^{2}}{t^{2}}\right)-\frac{t_{i}}{t}\right)
\end{gathered}
$$

where $\frac{N}{t_{i}^{3}}$ is the number density introduced in IIIB. It is evident from the last equation that $g\left(t_{i}, t_{i}\right)$. Let us identify in Equation (15) the two first terms of a series: $\frac{\mathrm{d} t}{\mathrm{~d} s}=\sum_{l=0}^{\infty} F^{(l)}(t)$ with $F^{(0)}(t)=1$ and

$$
F^{(1)}(t)=\frac{\pi G N m}{3 t_{i}^{3}} t^{2} g\left(t, t_{i}\right)
$$

The time evolution of the passive gravitational mass is $M(t)=m \alpha+m \frac{\mathrm{d} t}{\mathrm{~d} s}$; and, taking into account (15) can be expressed in the form:

$$
M(t)=m\left(1+\alpha+\frac{\pi G N m}{3 t_{i}^{3}} t^{2} g\left(t, t_{i}\right)+O\left(G^{2}\right)\right),
$$

that verifies $M\left(t_{i}\right)=m+m \alpha$. Let us recall that the constant $m \alpha$ introduced in Equation (13) was interpreted as the passive gravitational mass acquired during the epoch $a<a_{i}$. Multiplying (18) by the number density of particles we get the energy density valid for the recent universe, at redshift $z<z_{i}$ :

$$
\rho(t)=n(t) m\left(1+\alpha+\frac{\pi G N m}{3 t_{i}^{3}} t^{2} g\left(t, t_{i}\right)+O\left(G^{2}\right)\right) .
$$

\section{a) Construction of a cosmological model with gravitational mass}

As we are considering a model made exclusively of baryons, the term baryonic mass should refer to the constant inertial mass $m$ introduced in (2) and $\Omega_{b a}=\frac{8 \pi G}{3 H_{0}^{2}} n_{o} m$, be interpreted as the dimensionless baryonic density parameter.

The baryonic matter, as shown in Figure 2, is continuously acquiring gravitational mass (3) due to the gravitational interaction. To obtain the cosmological model, denoted above as Milne's universe with mass, we need to transform the harmonic coordinates used in section II into standard cosmological coordinates, but that is not so simple as was in the Milne's universe without mass treated in section IIIB. We shall assume now that $t(a)$ may be expressed as a series $t(a)=\sum_{k=0}^{\infty} t^{(k)}(a)$ (though we shall need only the first term $t^{(0)}$ ) and consider that:

1.It is verified $n(t(a))=\frac{n_{o}}{a^{3}}$, with $n_{o}$ equal to the number density of baryons 
at the present epoch. Let us recall that $\frac{N}{t_{i}^{3}}$ is the constant number density of particles over the hyperboloid $\tau_{i}=t_{i}$, introduced in IIIB, therefore we can write $\frac{N}{t_{i}^{3}}=\frac{n_{o}}{a_{i}^{3}}$.

b) The Equations (15) and (7), with $k=0$, determine the functions $t=t(s)$ and $s=s(a)$ up to first order in $G$.

First we identify in (15) the two first terms of the series $\frac{\mathrm{d} t}{\mathrm{~d} s}=\sum_{l=0}^{\infty} F^{(l)}(t)$, namely: $F^{(0)}(t)=1$, and $F^{(1)}(t)=\frac{\pi G N m}{3 t_{i}^{3}} t^{2} g\left(t, t_{i}\right)$. Then, from Equation (7) we have $\frac{\mathrm{d} s}{\mathrm{~d} a}=\frac{1}{H_{0} a}\left(\frac{\Omega_{M}}{a^{3}}+f(a)\right)^{-1 / 2}$ and using $\frac{\mathrm{d} t}{\mathrm{~d} a}=\frac{\mathrm{d} s}{\mathrm{~d} a} \frac{\mathrm{d} t}{\mathrm{~d} s}$ we obtain the differential equation that determines the series $t(a)=\sum_{l=0}^{\infty} t^{(l)}(a)$

$$
\frac{\mathrm{d} t}{\mathrm{~d} a}=\frac{1}{H_{0} a}\left(\frac{\Omega_{M}}{a^{3}}+\frac{\Omega_{b a}}{a^{3}} \sum_{l=1}^{\infty} F^{(l)}(t(a))\right)^{-1 / 2} \sum_{l=0}^{\infty} F^{(l)}(t(a)) .
$$

To order zero one obtains $\frac{\mathrm{d} t^{(0)}}{\mathrm{d} a}=\frac{1}{a H_{0}} \sqrt{\frac{a^{3}}{\Omega_{M}}}$, whose solution is

$$
H_{0} t^{(0)}(a)=\frac{2 a^{3 / 2}}{3 \Omega_{M}^{1 / 2}},
$$

and substituting (21) into (16) we have $g\left(t^{(0)}(a), t_{i}\right)=\tilde{g}\left(a, a_{i}\right)$ with

$$
\tilde{g}\left(a, a_{i}\right)=\left(1+\frac{a_{i}^{3}}{a^{3}}\right)^{3}-4 \frac{a_{i}^{3}}{a^{3}}\left(\frac{3}{2}\left(1+\frac{a_{i}^{3}}{a^{3}}\right)-\frac{a_{i}^{3 / 2}}{a^{3 / 2}}\right) .
$$

Using the prescription 1 given above we get $n(t(a)) m=\frac{3 H_{0}^{2}}{8 \pi G} \frac{\Omega_{b a}}{a^{3}}$, and taking into account (18) and (21) we obtain the dependence of the gravitational mass of a galaxy on the expansion factor, valid for the galactic dominance epoch $a>a_{i:}$

$$
M(a)=m\left(1+\alpha+\frac{G \Omega_{b a}}{18 \Omega_{M}} \frac{a^{3}}{a_{i}^{3}} \tilde{g}\left(a, a_{i}\right)\right) .
$$

It is manifest that the quotient $M(a) / m$ is independent of $m$, but not a constant. This fact prevents from identifying inertial and gravitational mass as quoted at the end of section II, but just this un-equality, far of been a drawback, is the clue to explain in the next section the origin of the cosmological dark components. Now, we can construct a cosmological model with dominant gravitational mass. By considering Equations (19) and (21) we obtain $\rho_{F}(a):=\rho(t(a))$, with

$$
\rho_{F}(a)=\frac{3 H_{0}^{2}}{8 \pi G} \frac{\Omega_{b a}}{a^{3}}\left(1+\alpha+\frac{\Omega_{b a}}{\Omega_{M}} \frac{a^{3}}{18 a_{i}^{3}} \tilde{g}\left(a, a_{i}\right)\right)+O\left(G^{2}\right)
$$

that can be written in the form (6) to identify $\Omega_{M}$ and $f(a)$ as follow:

$$
\rho_{F}(a)=\frac{3 H_{0}^{2}}{8 \pi G}\left(\frac{\Omega_{M}}{a^{3}}+f(a)\right)
$$




$$
\Omega_{M}=\Omega_{b a}(1+\alpha), f(a)=\frac{\Omega_{b a}^{2} \tilde{g}\left(a, a_{i}\right)}{18 \Omega_{M} a_{i}^{3}} .
$$

Our model has introduced three parameters: $\Omega_{b a}, \alpha, a_{i}$, but the identity $\Omega_{M}+f(1)=1$ gives a relation between them:

$$
\Omega_{b a}(1+\alpha)+\frac{\Omega_{b a} \tilde{g}\left(1, a_{i}\right)}{18(1+\alpha) a_{i}^{3}}=1 .
$$

The function $\tilde{g}\left(a, a_{i}\right)$ vanishes at $a=a_{i}$ and rapidly tends to unity for increasing a. From $\tilde{g}\left(a_{i}, a_{i}\right)=0$ we obtain, using Equations (23) and (25), that $M\left(a_{i}\right)=m(1+\alpha)$ and $\rho_{F}\left(a_{i}\right)=\frac{n_{o}}{a_{i}^{3}} m(1+\alpha)$; hence, we conclude that: at the beginning of the galactic epoch, $m \alpha$ is the gravitational mass contained in a galaxy, and $\frac{n_{o} m \alpha}{a_{i}^{3}}$ the gravitational mass density. Both were generated during the anterior epoch, with $a<a_{i}$, by the gravitational interaction of the particles that collapsed to form a galaxy.

4) Comparison of the model with gravitational mass with the $\Lambda \mathrm{CDM}$ cosmological model

The success of the $\Lambda C D M$ cosmological model has been corroborated by the Planck 2013 results [17]. This model considers null pressure and introduces the cosmological constant $\Lambda$, by substituting $f(a)=\Lambda / 3 H_{0}^{2} \equiv \Omega_{\Lambda}$ into Equation (6), to get $\rho_{F}=\frac{3 H_{0}^{2}}{8 \pi G}\left(\frac{\Omega_{M}}{a^{3}}+\Omega_{\Lambda}\right)$. The Equations (8) and (9) imply a constant negative pressure and a variable acceleration: $p=-\frac{3 H_{0}^{2}}{8 \pi G} \Omega_{\Lambda}, \frac{\ddot{a}}{a}=-\frac{H_{0}^{2}}{2}\left(\frac{\Omega_{M}}{a^{3}}-2 \Omega_{\Lambda}\right)$. The density parameter $\Omega_{M}$ contains baryon and dark matter contributions: $\Omega_{M}=\Omega_{b a}+\Omega_{d m}$. The model works well, fitting the observed supernovae moduli-distance redshift relation [18], and the unexpected recent transition from decelerated to accelerated universe at redshift $z=0.6$.

However, the problematic physical interpretation of the cosmological constant as vacuum energy density, caused the introduction of the new physical field dubbed dark energy. A comprehensive review of the new dark components can be found in the books [19] [20]. Assuming $h=0.67$ for the reduced Hubble constant, one gets estimations for the density fractions: $\left(\Omega_{b a}, \Omega_{d m}, \Omega_{\Lambda}\right)=(0.049,0.268,0.683)$.

Let us give our interpretation of the dark matter parameter $\Omega_{d m}$ in the $\Lambda C D M$ model: our model use the parameter $\Omega_{M}=\Omega_{b a}(1+\alpha)$, where we have substituted $\Omega_{b a} \alpha$ in place of $\Omega_{d m}$. Accordingly to the interpretation of $m \alpha$ at the end of the anterior section, $\frac{n_{o}}{a_{i}^{3}} m \alpha \equiv \frac{3 H_{0}^{2}}{8 \pi G} \frac{\Omega_{b a} \alpha}{a_{i}^{3}}$ is the gravitational mass density at the beginning of the galactic dominance epoch, acquired by the particles dominant in the precedent epoch with $a<a_{i}$.

Finally, although we will not use the cosmological constant as parameter, in the next section we shall give a physical interpretation of this constant as the asymptotic value of the gravitational mass density. 


\section{Gravitational Mass Density in the Galactic Dominance Epoch: An Interpretation of Dark Matter and Dark Energy}

The objective of this section is to show that the notion of gravitational mass, introduced in this paper to construct the Milne's universe with mass, affords a satisfactory explanation, at cosmological scales, of both dark components.

1) Gravitational mass instead of dark matter, cosmological constant, and dark energy

The gravitational mass fraction $f(a)$ obtained in (26) depends of three parameters $\left(\Omega_{b a} \alpha, a_{i}\right)$ constrained by the Equation (27). We can obtain good values for them identifying our parameter $\Omega_{M}=\Omega_{b a}(1+\alpha)$ with the equivalent in the $\Lambda C D M$ model $\Omega_{M}=\Omega_{b a}+\Omega_{d m}$ described in IIID, obtaining the relations $\Omega_{b a}=0.049$, and $\Omega_{b a} \alpha=\Omega_{d m}=0.268$ and from the last one we get $\alpha=\frac{\Omega_{d m}}{\Omega_{b a}}=5.47$. To determine the remaining parameter $a_{i}$ we observe that, as it is shown in Figure $3, f(a)$ is a monotonous increasing function of the expansion factor, verifying $f\left(a_{i}\right)=0$, that rapidly tends to a constant value $f(1)$. If we identify

$f(1)=\Lambda / 3 H_{0}^{2}=\Omega_{\Lambda}=0.683$ we obtain the equation $\frac{g\left(1, a_{i}\right)}{a_{i}^{3}}=\frac{18 \Omega_{M} \Omega_{\Lambda}}{\Omega_{b a}^{2}}$, that determines the beginning of the galactic epoch at $a_{i}=0.085$, that corresponds to redshift $z_{i}=10.76$. This is our explanation of the cosmological constant $\Lambda$ as a limit value of the gravitational mass density introduced in this paper, that makes unnecessary to surmise the existence of a new physical field, so-called dark-energy. This is a physical interpretation of the cosmological constant $\Lambda$ as the limit of the gravitational mass density. This interpretation of the constant $\Omega_{\Lambda}$ has no problem, unlike the dark energy assumption, with the coincidence of the densities $\frac{\Omega_{m}}{a^{3}}$ and $f(a)$ at a so recent epoch as $z_{i}=0.3$ [20], because in our

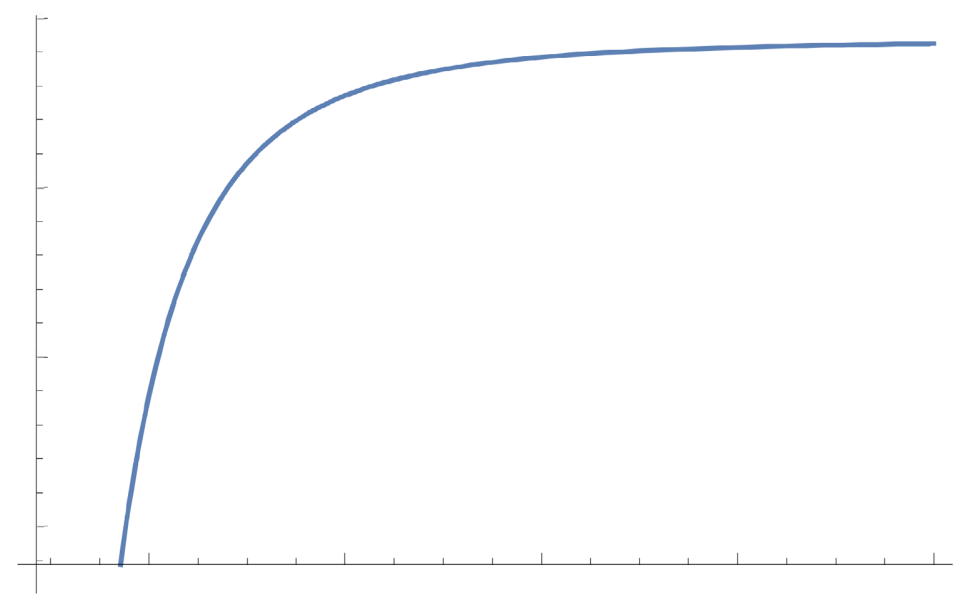

Figure 3. The gravitational mass fraction as function of the expansion factor. The gravitational mass fraction $\left(f(a)=\right.$ gravitational mass density $\left./ \rho_{c r i}\right)$ of our model is a function of the expansion factor $a$. In a neighbourhood of $a=1$ the function $f(a)$ is practically constant. 
case, $\frac{\Omega_{M}}{a^{3}}$ and $f(a)$ should not be considered as two unrelated magnitudes, rather they are two components of a sole gravitational mass density. Though $f\left(a_{i}=0\right), f(a)$ increases rapidly, the $\frac{\Omega_{m}}{a^{3}}$ is a decreasing function, and the graphs of both components cross for $a_{c}=\frac{1}{1.3}$.

In the next subsections we show how using the values $\left(\Omega_{b a}, \alpha, a_{i}\right)=(0.049,5.47,0.085)$ obtained in this section, one can reproduce with great exactitude the main predictions of the $\Lambda C D M$ model.

\section{2) The moduli-distance redshift relation}

With the gravitational mass density $f(a)$ we can explain the luminosity distance $d_{L}(z)$, or the equivalent logarithmic moduli-distance

$\mu(z)=5 \log d_{L}(z)+25$, to a source with redshift $z$. The difference between our prediction $\mu_{f}(z)$ and the $\Lambda C D M$ prediction $\mu_{\Lambda}(z)$ for the supernovae observations accounted in [18] is of the order of $10^{-5}$. The discrepancy, $\frac{\mu_{f}(z)-\mu_{\Lambda}(z)}{\mu_{\Lambda}(z)}$, between both models is represented in Figure 4.

\section{3) The accelerated universe}

With the Equation (9) without cosmological constant it is impossible to explain an accelerated universe, unless the pressure of the cosmological fluid be negative, but an important consequence of the gravitational interaction between the particles is that the gravitational mass density, $f(a) \frac{3 H_{0}^{2}}{8 \pi G}$, given in (26), when substituted into the Equations (8) and (9), gives the necessary negative pressure. We can conclude that the gravitational pressure is the cause of the

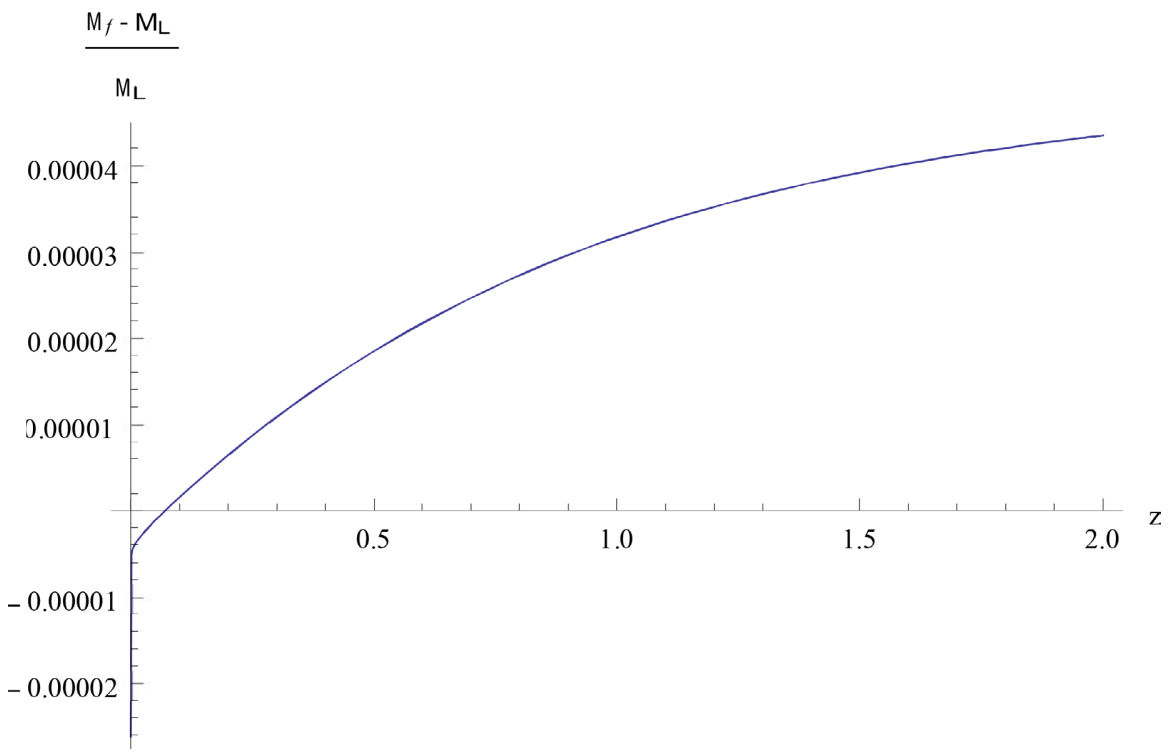

Figure 4. Our prediction of the moduli-distance redshift relation about the supernovae observations [18], is practically indistinguishable from the one based on the $\Lambda C D M$ cosmological model. 
acceleration of the universe. As it is shown in Figure 5, the deceleration factor $q=-\ddot{a} a / \dot{a}^{2}$ becomes negative recently because $f(a)$ corresponds to the galactic dominance epoch with expansion factor $a_{i}<a<1$.

\section{4) The pressure to density ratio $w(a)$}

Let us decompose in two summands the energy density given in (25), (26): $\rho_{F}(a)=\rho_{M}(a)+\rho_{f}(a)$, with $\rho_{M}(a)=\frac{3 H_{0}^{2}}{8 \pi G} \frac{\Omega_{M}}{a^{3}}$ and $\rho_{f}(a)=\frac{3 H_{0}^{2}}{8 \pi G} f(a)$. As only the component $\rho_{f}(a)$ contributes to the pressure given in (8), it is worthwhile to know our prediction for the ratio $w_{f}(a)=\frac{p(a)}{\rho_{f}(a)}$. Using (8) one gets:

$$
w(a)=\frac{a}{3} \frac{\mathrm{d} \ln f}{\mathrm{~d} a}-1 .
$$

In Figure 6 we have represented the function $w(a)$ predicted by our model. It is manifest a linear dependence for values close to unity (very low redshifts) and a rapid increase when a decreases (for high redshifts).

The variability of the ratio $w(a)$ has been tested in [21] under a linear dependence hypothesis: $w(a)=w_{0}+(1-a) w(a)$, obtaining the constraint $-1.33<w_{0}<-0.79$ for the present value $w_{0}=w(1)$ of the pressure density ratio. With equation (28) we get $w(1)=-0.998$ that satisfies the constraint, but the linear dependence in our prediction, showed in Figure 6, clearly fails for $a<0.4 \quad(z>1.5)$. Let us remark that in the today vast literature on dark energy one has introduced, besides the scalar field language with the quintessences, some barotropic fluid models as origin of the acceleration [22], whose $w($ a) ratio are qualitatively similar to our prediction. The pressure given in (8) has dynamic

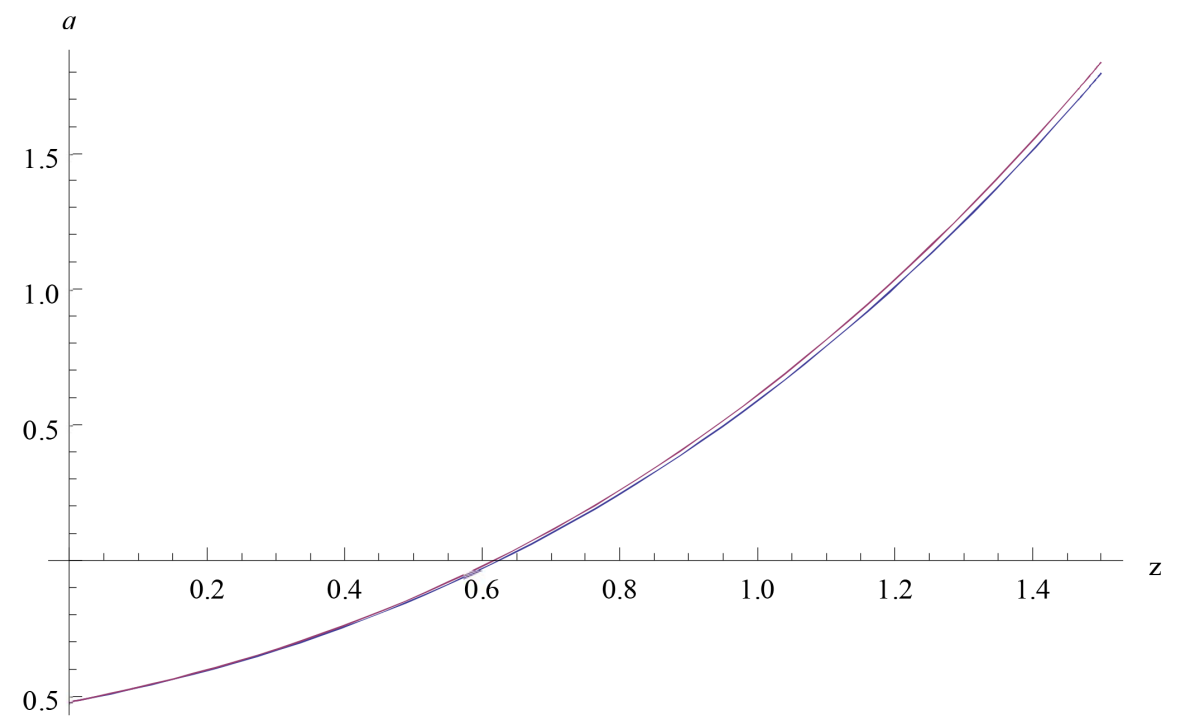

Figure 5. The deceleration factor $q$ becomes negative recently at redshift 0.6 . The upper curb corresponds to our model, as a consequence of the gravitational energy density $f(a)$, the lower one is the prediction made by the $\Lambda C D M$ model based on the assumption of a cosmological constant. 


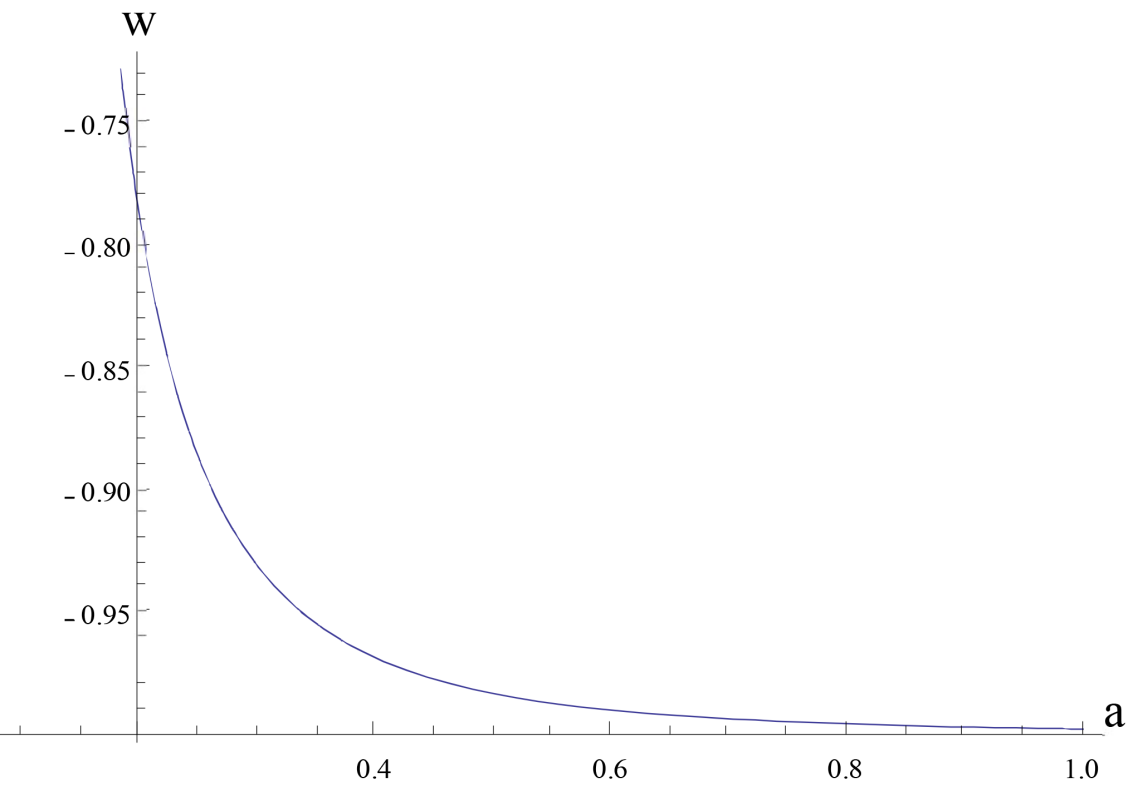

Figure 6. This figure shows the pressure to density ratio $w(a)=p(a) / \rho_{\ell}(a)$ predicted by our model for the galactic dominance epoch, $\frac{1}{11.7} \leq a \leq 1$.

character rather than kinetic, because it is linked to the gravitational energy density $f(a)$ described in section IVA. The potential energy contribution to a dynamic pressure is well known in classical mechanics, see Chp.17.2 in [23] and Chp. 7.4 in [24], but little is known about that in general relativity.

\section{5) Time evolution of the gravitational mass fraction}

A very interesting result comes from Equation (23), that describes the dependence of the gravitational mass of a point-like particle on the expansion factor. We can estimate the evolution of the gravitational mass fraction of a generic galaxy with the expression

$$
f_{G M}(a)=\frac{M(a)-m(1+\alpha)}{M(a)} .
$$

Our interpretation of dark matter as the gravitational mass acquired by the particles that collapsed to form a galaxy implies that we must compare $f_{G M}(a)$ with dark matter observations at different redshifts. The observed redshift dependence of the dark matter fraction $f_{D M}(z)$ can be accounted for with the function $f_{G M}(a)$, after substituting $a=\frac{1}{1+z}$, showed in Figure 7 as a continuous curve. The two first fractions were observed by Dutton [25] in 2011 and Suyu [26] in 2012, and the last six, correspond to more recent observations by Gencel et al. [27].

\section{Conclusions}

We have used P. Havas and J.N. Goldberg results on dynamics of a finite number of gravitating point-like particles to revise the concepts of inertial and gravitational 


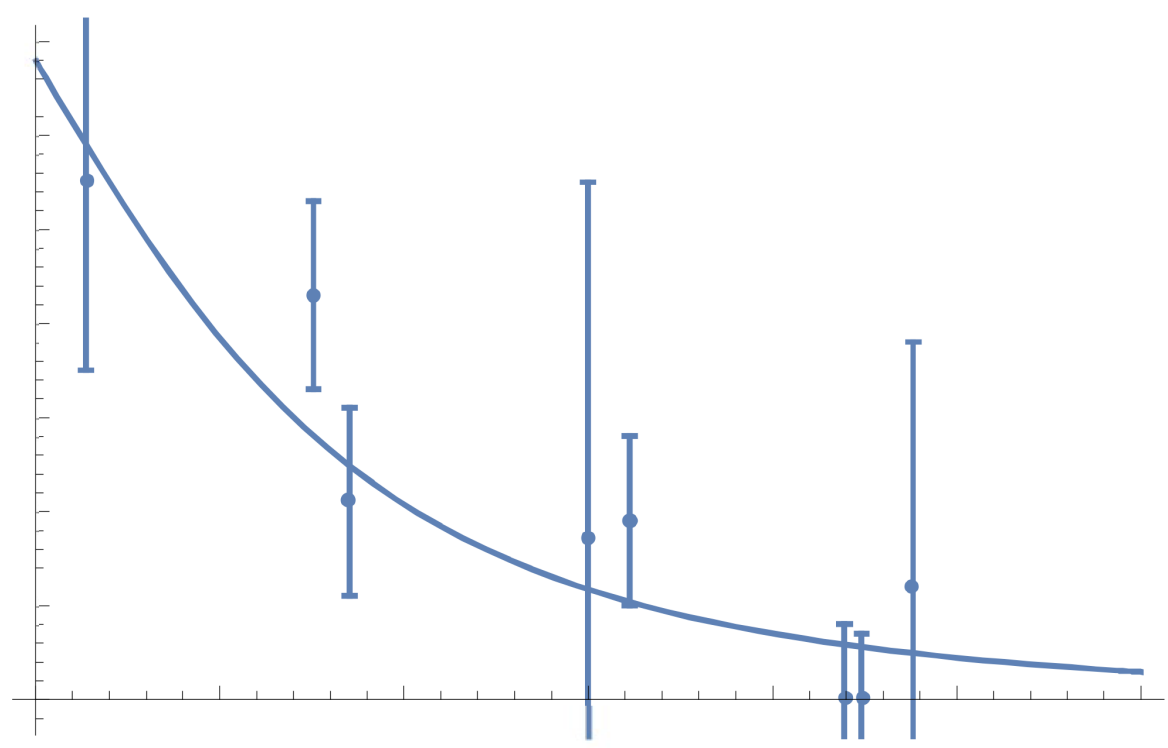

Figure 7. The gravitational mass fraction $f_{G M}(z)$ as function of the redshift calculated with our model for point-like particles fits well to a sample of observed values in different galaxies up to redshifts of the order 2 .

mass in general relativity and derive their cosmological consequences:

a) Inertial and gravitational mass. The law of motion of a particle (5) suggests definitions for a constant inertial mass, $m$, and a passive gravitational mass, $M=m / \sqrt{g(v, v)}$, depending on the metric generated by the particles. In section IIIC, we proved the equivalence of passive and active gravitational mass, and obtained its monotonous increasing dependence on the expansion factor $M(a)$. In Figure 2, we show how the excess of gravitational mass, $M(a)-m$, at an event $P$ on a world line $L$ is due to the gravitational interaction with the world lines intersecting its past light cone. When time passes, the number of intersected lines, and $M(a)$, increases.

b) Dark matter, dark energy and cosmological constant as different aspects of the gravitational mass. In section IVA, we have estimated the beginning of the galactic dominance at $a_{i}=0.085 \quad\left(z_{i}=10.76\right)$. The gravitational mass fraction $f(a)$, showed in Figure 3, verifies $f\left(a_{i}\right)=0$ and is practically a constant in the interval $0.4<a \leq 1$. The value $f(1)$ is identified with the cosmological constant. We have reinterpreted the $\Lambda C D M$ density parameters $\left(\Omega_{d m}, \Omega_{\Lambda}\right)=(0.268,0.683)$ in terms of gravitational mass: $\Omega_{d m}$ is now the gravitational mass density, $\frac{8 \pi G}{3 H_{0}^{2}} n_{o} m \alpha$, acquired by the dominant particles before the galactic era; $\Omega_{\Lambda}$ is the present value, $f(1)$, of the time dependent gravitational mass density. Therefore, in our model, the universe is roughly formed by $5 \%$ of baryonic and $95 \%$ of gravitational mass: a $27 \%$, usually referred as dark matter, was acquired during the epoch $a<a_{i}$ previous to the galactic dominance, and a $68 \%$, usually described as dark energy, is the gravitational mass generated in the galactic dominance era $a>a_{i}$. This interpretation is free of the coincidence problem [20]: the value of $\Omega_{\Lambda}$ is close to $\Omega_{M}=\Omega_{b a}+\Omega_{d m}$, because having a common origin, they are not unrelated mag- 
nitudes.

c) Relation distance-redshift. As showed in Figure 4, we have obtained the moduli-distance as function of the redshift that explains the supernova observations. It is indistinguishable from the one obtained with the $\Lambda C D M$ model using a cosmological constant.

d) The accelerated universe. The pressure of the non ideal gas filling the universe given in (8) is not kinetic. It is a functional of the gravitational mass density $\rho_{f}(a)=f(a) \frac{3 H_{0}^{2}}{8 \pi G}$. This pressure is liable of the recent acceleration of the universe: substituted in (9) produces the deceleration factor $q$ showed in Figure 5.

e) The equation of state of the cosmological fluid. We have obtained the equation of state of the cosmological fluid by the pressure to density ratio $w(a)=\frac{p(a)}{\rho_{f}(a)}$ given in (28) and shown in Figure 6, satisfying the observational constraint $-1.33<w(1)<-0.79$.

f) Time evolution of the gravitational mass fraction. We have given the time evolution of the gravitational mass of a point-like particle in Equation (23) and the consequent gravitational mass fraction (29). They are in good agreement, as shown in Figure 7, with the mass measurements of galaxies up to redshift $z \sim 2$, usually reported as dark matter fractions.

We conclude that the gravitational energy density introduced in this paper explains the large scale cosmological observations in the galactic dominance epoch, making unnecessary either the cosmological constant or the dark matter and energy assumptions. To extend the analysis of the gravitational mass to an inhomogeneous universe is more complicated, but necessary to predict its distribution inside the galaxies.

\section{Acknowledgements}

This work has been supported by the Spanish "Ministerio de Economía y Competitividad" and the "Fondo Europeo de Desarrollo Regional" MINECO-FEDER Project No. PGC2018-095251-B-100, and by the Spanish Ministerio de Ciencia, Innovación y Universidades and the Fondo Europeo de Desarrollo Regional, Projects PID2019-109753GB-C21 and PID2019-109753GB-C22, the Generalitat Valenciana Project AICO/2020/125 and the University of Valencia Special Action Project UV-INVAE19-1197312.

\section{Conflicts of Interest}

The author declares no conflicts of interest regarding the publication of this paper.

\section{References}

[1] Jammer, M. (2000) Concepts of Mass in Contemporary Physics and Philosophy. Princeton University Press, Princeton. https://doi.org/10.1515/9781400823789 
[2] Havas, P. and Goldberg, J.N. (1962) Physical Review, 128, 398. https://doi.org/10.1103/PhysRev.128.398

[3] Fock, V. (1959) The Theory of Space Time and Gravitation. Pergamon Press, Oxford, 342-352.

[4] Belifante, F.J. (1962) Physical Review, 125, 1124. https://doi.org/10.1103/PhysRev.125.1124

[5] Bel, L., Damour, T., Deruelle, N., et al. (1981) General Relativity and Gravitation, 13, 963-1004. https://doi.org/10.1007/BF00756073

[6] Damour, T. (1982) Gravitational Radiation and the Motion of Compact Bodies in Gravitational Radiation. North-Holland, Amsterdam.

[7] Geroch, R. and Traschen, J. (1962) Physical Review, 36, 1124.

[8] Katanaev, M.O. (2013) General Relativity and Gravitation, 45, 1861-1875. https://doi.org/10.1007/s10714-013-1564-3

[9] Beltran, P. and Portilla, M. (2021) The Extended Hyperbolic Einstein-Rosen Bridge.

[10] Portilla, M. (2015) Journal of Physics: Conference Series, 600, Article ID: 012007. https://doi.org/10.1088/1742-6596/600/1/012007

[11] Landau, L.D., Akhiezer, A.I. and Lifschitz, E.M. (1967) General Physics, Mechanics and Molecular Physics. Pergamon Press, Oxford. https://doi.org/10.1016/B978-0-08-009106-8.50004-2

[12] Einstein, A. (1950) The Meaning of Relativity. 4th Edition, Methuen, London.

[13] Brans, C.H. (1962) Physical Review, 125, 388. https://doi.org/10.1103/PhysRev.125.388

[14] Rosen, N. (1965) Annals of Physics, 35, 426-436. https://doi.org/10.1016/0003-4916(65)90248-4

[15] Ohanian, H.C. (2013) The Energy-Momentum Tensor in General Relativity and in Alternative Theories of Gravitation, and the Gravitational vs. Inertial Mass. https://arxiv.org/abs/1010.5557

[16] Rindler, W. (2006) Relativity, Special, General, and Cosmological. 2th Edition, Oxford University Press, Oxford.

[17] Planck Collaboration (2014) Astronomy and Astrophysics, 571, A16.

[18] Riess, A.G., et al. (2007) The Astrophysical Journal, 659, 98. https://doi.org/10.3139/146.070802

[19] Peebles, P.J.E. (1993) Principles of Physical Cosmology. Princeton University Press, Princeton.

[20] Amendola, L. and Tsujikawa, S. (2010) Dark Energy. Theory and Observations. Cambridge University Press, Cambridge. https://doi.org/10.1017/CBO9780511750823

[21] Komatsu, E., et al. (2009) The Astrophysical Journal, 180, 330. https://doi.org/10.1088/0067-0049/180/2/330

[22] Goodstein, D. (2012) Adventures i Cosmology. World Scientific, Singapore. https://doi.org/10.1142/7820

[23] Hill, T.L. (1986) An Introduction to Statistical Thermodynamics. Dover Publications Inc., Mineola.

[24] Kreuzer, H.J. (1981) Nonequilibrium Thermodynamics and Its Statistical Foundations. Oxford Science Publications, Oxford.

[25] Dutton, A.A., et al. (2011) Monthly Notices of the Royal Astronomical Society, 417, 
1621.

[26] Suyu, S.H., et al. (2012) The Astrophysical Journal, 750, 10. https://doi.org/10.1088/0004-637X/750/1/10

[27] Genzel, R., et al. (2017) Nature, 543, 397. 